\title{
A Case Series Demonstrating a Novel Technique for Reversal of Trabeculectomy Using Lamellar Sclerectomy and Corneal
}

\section{Graft}

\author{
Saaquib R Bakhsh ${ }^{1}$, David Rooney ${ }^{2}$, Bryan A Goldman ${ }^{3}$, Tom Obertynski ${ }^{4}$, Chirag K Gupta ${ }^{5}$
}

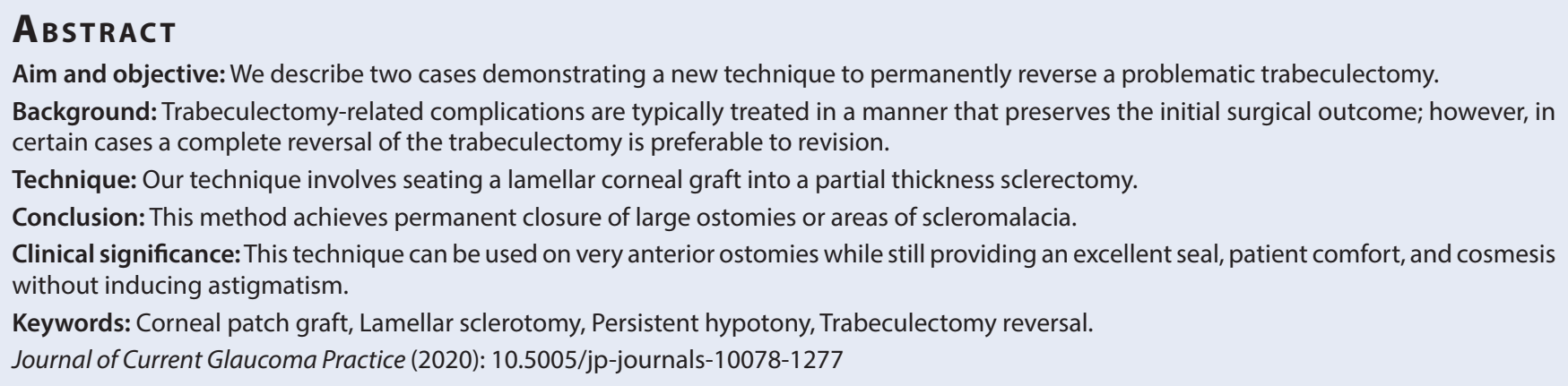

\section{INTRODUCTION}

Trabeculectomy has long been considered the gold standard in the surgical management of glaucoma. Despite the overall success and prevalence of trabeculectomy, the surgery confers numerous potentially severe short- and long-term complications, including diplopia ${ }^{1}$ and motility disturbances, ${ }^{2}$ hypotony, dysesthesia, and endophthalmitis. ${ }^{3} \mathrm{~A}$ variety of techniques have been developed to manage trabeculectomy-related complications and preserve trabeculectomy function. However, in certain circumstances a trabeculectomy reversal may be preferable to revision.

We describe two cases demonstrating a novel surgical technique for the reversal of problematic trabeculectomies. We discuss the preoperative decision-making process and the postoperative course of a 64-year-old woman with primary openangle glaucoma (POAG) with a symptomatic overhanging bleb and a 52-year-old-man with POAG and persistent hypotony from an overfiltering trabeculectomy who underwent this procedure.

\section{Case Descriptions}

\section{Case 1}

A 64-year-old woman with a history of long-standing POAG initially treated with trabeculectomy using 5-fluorouracil in the right eye 22 years prior, presented with constant irritation and double vision. The patient's examination was notable for an oversized, avascular, cystic bleb of the right eye overhanging the cornea and inducing irregular astigmatism. A scleral trap door was absent and there was a large area of scleromalacia extending anteriorly near the limbus. The patient's severe bleb-induced dysesthesia and astigmatism were refractory to conservative treatments, and the patient elected to undergo trabeculectomy reversal.

\section{Surgical Technique}

Adequate anesthesia and akinesia were achieved with a retrobulbar block, the superior bleb was excised, and a 4-mm corneal trephine
1,2,5 Department of Ophthalmology, Beaumont Eye Institute, Royal Oak, USA

${ }^{3}$ Department of Ophthalmology, Virginia Commonwealth University, Richmond, Virginia, USA

${ }^{4}$ Department of Ophthalmology, Beaumont Eye Institute, Royal Oak, USA; Michigan Glaucoma Specialists, Royal Oak, Michigan, USA

Corresponding Author: Chirag K Gupta, Department of Ophthalmology, Beaumont Eye Institute, Royal Oak, USA, Phone: +1 248-551-2020, e-mail: Chirag.Gupta@beaumont.org

How to cite this article: Bakhsh SR, Rooney D, Goldman BA, et al. A Case Series Demonstrating a Novel Technique for Reversal of Trabeculectomy Using Lamellar Sclerectomy and Corneal Graft. J Curr Glaucoma Pract 2020;14(2):61-63.

Source of support: Nil

Conflict of interest: None

was then used to encompass the atrophic area. A lamellar dissection of the sclera was performed, and a lamellar corneal graft was prepared using a 5-mm corneal trephine. The graft was sutured into the partial-thickness scleral hole, resulting in a water-tight seal. Conjunctiva was mobilized and sutured to the limbus to cover most of the exposed sclera and corneal graft. Amniotic membrane was then placed over the remaining exposed area, glued with fibrin sealant, and anchored to the limbus, and sub-conjunctival antibiotics and steroids were administered.

\section{Case 2}

A 52-year-old man with a history of POAG initially controlled for two years with a trabeculectomy using mitomycin $C$ subsequently required two revisions for hypotony including one scleral patch graft. He later presented with constant tearing and was found to have a leaking and overfiltering bleb of the right eye. Conservative measures including the use of a large diameter bandage contact 


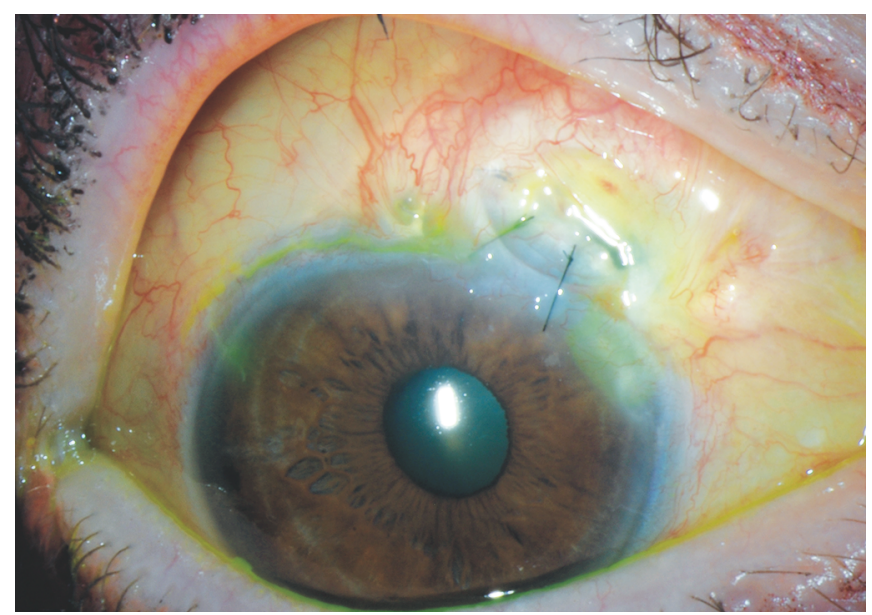

Fig. 1: A 10-month postoperative image of the first case, demonstrating a well-seated and low-profile graft in the previous trabeculectomy site

lens did not result in self-sealing of the surgical site and the patient elected to undergo trabeculectomy reversal.

\section{Surgical Technique}

A peritomy was made with Westcott scissors and forceps. The initial scleral patch graft was removed, and the superior ostomy was found to be full thickness with thin sclera. A 3.0-mm area of three-quarter depth was excavated around the superior ostomy, and $4.0-\mathrm{mm}$ button of donor corneal tissue was sutured into place creating a water-tight seal. The conjunctiva was then sutured to the graft, and an amniotic membrane was placed over the graft and under the conjunctiva and glued into place, covering the graft completely. Finally, steroid and antibiotic ointment were placed in the eye.

\section{Results}

In both cases, the corneal patch graft remained in place, and the wound was Seidel negative with conjunctiva covering the graft one week after surgery. Three months postsurgery, neither patient had pain, irritation, or leakage of aqueous from the eye. The monocular diplopia had resolved in the first case and the hypotony had resolved in the second. Neither patient had any significant induced astigmatism on corneal topography. The follow-up for the next year demonstrated excellent comfort and cosmesis for our first patient (Fig. 1) and normal IOP without aqueous leak or hypotony in our second patient (Fig. 2).

\section{Discussion}

Many surgical techniques have been developed to revise problematic trabeculectomy blebs. In 1979, Scheie and Guehl recommended managing symptomatic overhanging blebs by bluntly dissecting the excess conjunctiva off of the cornea, excising it with scissors, and suturing the bleb closed. ${ }^{4} \mathrm{~A}$ similar sutureless approach has since been described ${ }^{5}$ as have conjunctival advancement, flap, ${ }^{6}$ and cryopexy ${ }^{7}$ techniques with and without complete excision of the dysfunctional bleb. ${ }^{8,9}$ However, the longterm success of these techniques is variable. One case series that evaluated trabeculectomy revisions found that when performed for dysesthesia, only $57 \%$ of revisions were successful at a mean follow-up of 2.8 years. ${ }^{10}$ The study demonstrated that $21 \%$ $(6 / 28)$ of the patients experienced a recurrence or persistence of

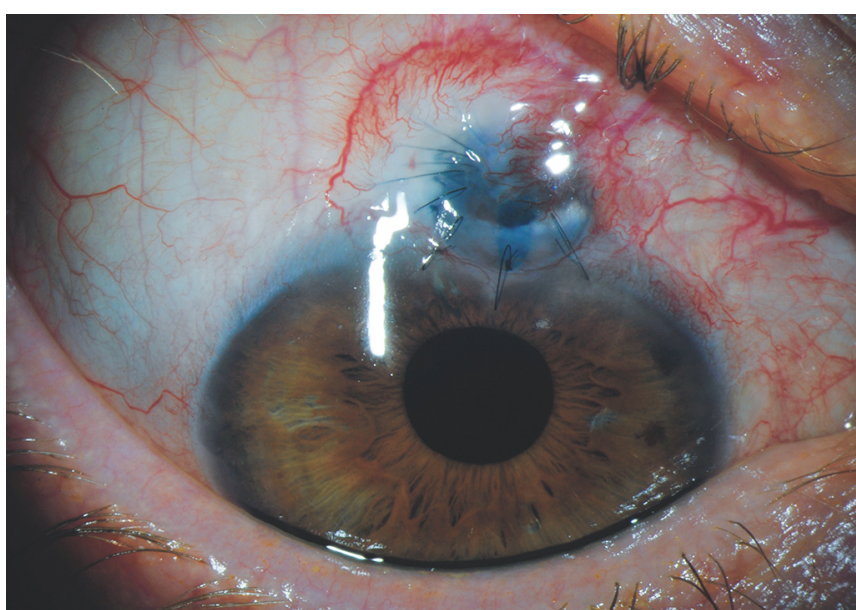

Fig. 2: A 9-month postoperative image of the second case, demonstrating a water-tight reversal of the previous trabeculectomy

dysesthesia, and the surgery for any indication yielded no significant improvement in visual acuity.

While we considered various revision approaches, our patients did not stand to benefit from revision by conjunctival advancement due to the brisk flow of aqueous and active leakage. In the first case, the reduction of the aqueous flow via revision of the scleral flap was impossible because of the extensive scleromalacia. Importantly, our patient showed no visual field loss on perimetry two decades after initial POAG diagnosis indicating a low risk of progression with postoperative increase in IOP. Furthermore, many medical and surgical options for the management of POAG have emerged since the patient's trabeculectomy over 20 years ago.

In both cases, due to the sclerostomy's anterior location, a corneal patch graft was deemed a better option than a scleral patch graft for achieving the desired appearance and comfort. Seating the lamellar graft into a partial thickness scleral dissection lowered the graft's profile, which further reduced the risk of localized dryness, astigmatism, and poor cosmesis. Amniotic membrane was used in the first case to ensure complete coverage of the exposed ocular surface because the large size of the bleb obviated direct conjunctival closure.

\section{Conclusion and Clinical Significance}

We recommend the consideration of our surgical technique for the patients with severe trabeculectomy-related complications who also have reasonable alternatives for the management of their glaucoma. While our encouraging results are limited to two patients, they suggest that this technique provides a safe, effective, and cosmetically acceptable reversal of problematic trabeculectomies.

\section{References}

1. Sun PY, Leske DA, Holmes JM, et al. Diplopia in medically and surgically treated glaucoma patients. Ophthalmology 2017;124(2):257-262. DOI: 10.1016/j.ophtha.2016.10.006.

2. Rauscher FM, Gedde SJ, Schiffmann JC, et al. Motility disturbances in the tube versus trabeculectomy study during the first year of follow-up. Am J Ophthalmol 2009;147(3):458-466. DOI: 10.1016/j. ajo.2008.09.019.

3. Yap ZL, Chin Y, Ku J, et al. Bleb related infections: clinical characteristics, risk factors, and outcomes in an Asian population. Clin Ophthalmology 2016;10:2303-2309. DOI: 10.2147/OPTH.S118809. 
4. Scheie HG, Guehl JJ. Surgical management of overhanging blebs after filtering procedures. Arch Ophthalmol 1979;97(2):325-326. DOI: 10.1001/archopht.1979.01020010171019.

5. Anis S, Ritch R, Shihadeh W, et al. Sutureless revision of overhanging filtering blebs. Arch Ophthalmol 2006;124(9):1317-1320. DOI: 10.1001/ archopht.124.9.1317.

6. Laspas P, Culmann PD, Grus FH, et al. Revision of encapsulated blebs after trabeculectomy: long term comparison of standard bleb needling and modified needling procedure combined with transconjunctival scleral flap sutures. PLOS One 2017;12(5):e0178099. DOI: 10.1371/journal.pone.0178099.
7. El-Harazi SM, Fellman RL, Feldman RM, et al. Bleb window cryopexy for the management of oversized, misplaced blebs. J Glaucoma 2001;10(1):47-50. DOI: 10.1097/00061198-200102000-00009.

8. La Borwit SE, Quigley HA, Jampel HD. Bleb reduction and bleb repair after trabeculectomy. Ophthalmol 2000;107(4):712-718. DOI: 10.1016/ s0161-6420(99)00174-8.

9. Lee GA, Holcombe DJ. Surgical revision of dysfunctional filtration blebs with bleb preservation, sliding conjunctival flap and fibrin glue. Eye 2010;24(6):947-953. DOI: 10.1038/eye.2009.279.

10. Radhakrishnan S, Quigley HA, Jampel HD, et al. Outcomes of surgical bleb revision for complications of trabeculectomy. Ophthalmo 2009;116(9):1713-1718. DOI: 10.1016/j.ophtha.2009.04.003. 